\title{
Light and Shadow Shape about Concept Art in Game
}

\author{
Yuqing $\mathrm{Ji}$ \\ Department of Animation College, Hebei Institute of Fine Art ,Shijiazhuang Hebei, 050700, China
}

Keywords: Light shape, Shadow shape, Modeling Game, Concept art

\begin{abstract}
This paper focuses on important modeling technique of expression of the design of the original game, at the end of this article summarizes some about light and shadow in the game concept design modeling method. Study discusses the modeling of light and shadow, and hope to dig deeper game the connotation of the original painting design. Thus in order to promote the development of the visuals of the game design field to do a little, inappropriate content, please the teacher and the game predecessors comment.
\end{abstract}

\section{Introduction}

Game development cannot be separated from the art people's cooperation, art images is very important for the subsequent development of the game as a whole. As most early and forefront of making parts, they were the first to come into contact with the entire game world to view the first batch of personnel, planning personnel art idea mainly dependent on the implementation of the original painting. In the original painting, props, scenes and characters are through in-depth communication with planning additional game art creators of creative to finish in the end, moreover, follow-up of $3 \mathrm{~d}$ production is mainly done through the concept design, finally completed the final image is introduce into the game big view of the world, art part of the game is completed. The development of computer science and technology is closely related to the development of the visuals of the game, is the rapid development and popularization of computer, computer prompted an increasing number of potential users, and the pursuit of better quality, visuals of the game is actually a combination of painting and art design. The common computer great creation function, a large number of the usage of material, comfortable and convenient environment and efficient design process, prompting visuals of the game rapid development, unlimited technique, delicate and exquisite picture, unique artistic characteristics of the artistic charm is unique to the visuals of the game. [1]

The visuals of the game screen tone, light and shadow is one of the basic factors affecting the modeling, but it is the most intuitive. Light is often used in the original painting creation of the game, light and shadow have a larger impact on the image, affects the smooth of the picture, the appeal of emotion, image and so on, light and shadow to form shape is worthy of further research. In addition, the modeling of light and shadow is often overlooked in the design of the original game is an important element, it takes the form of light and shade layout to metaphor object of the game's strong sense of volume and picture. "Images of all present items need to be combined with the Angle of the light to be reconciled." Shadow "picture, with the continuous improvement of image quality is being game art research and development team, the head of the attention, because the light and shadow model directly affects the quality of the picture. Light and shadow in the modeling, the dark can nourish the picture restless mood, can be a game players feel the space of the extrusion and deep. Use the form of light and shadow, dynamic and static, weight, and so on change human visual concepts, create the aesthetic of a new kind of high level, enrich the image modeling, added new vitality for art, concept should be a game designer constantly explore the pursuit of goals. Shadow form the boundary problem of images is the strength of the light and the effect of permeability, in real life, in addition to the sun and other industrial sources, basically belongs to soft light. Light is downy usually were widely used in CG the traditional painting and 
modern painting. [2]Visuals of the game in the design of light use is an important branch of the modeling of light and shadow. Light is divided into five main tone, specular, reflection and transition color, backlight, projection, these characteristics is our game original painting creation will use light and shadow in the modeling of expression, because the quality of the game is constantly improving, delicate degree is constantly improve, light soft degree to a great extent, affect the details of the visuals of the game, how to grasp the exquisite and soft light, visuals of the game is an important factor to consider in literary creation.

In today's teaching and industry of game of the original painting, most important is by compliant or extensive expressive line, strong and hazy shadow and color mutual collocation ability reflected the, so in the performance of the original painting we want to put many of these elements are combined and synthetic physical distribution and utilization in order to reflect the good artistic effect. The first step, the first to understand the light. Physics is the interpretation of the meaning of light, light is reflected by the expression of a kind of electromagnetic wave and the way of communication, it is a kind of virtual communication means to carry out the performance of the. Physics is called "the two wave of". Game design, use of light and shadow style is reflected in where? The common meaning, because the light we can see real natural color, high light in Western painting art is refers to the object itself in the brightest point, after blocking the light, the surrounding light, and the object itself no shadow of, light shadowless body feeling bad, can think, Western painting art refers to the light is in fact our popular parlance of light and shade.

Light is divided into five tone, high gloss, color transition, backlight, reflection and projection, these features is often used in our game painting creation of light and shadow shapes, because the quality of the game in continuous improvement, delicate picture degree in continuous improvement, soft light and in a great extent affects the details of the original game concept, how to grasp these delicate and soft lighting performance, is one of the most important factors need to be considered in the creation of the original games.

\section{Soft Light Performance}

The boundary problem of light and shadow forms in the picture, can reflect the intensity and the permeability of light, in reality, in addition to the sun other industrial light source, all belong to soft light source. In the same time, soft is usually widely used in traditional painting and modern CG painting. An important branch of the use of light and shade in the original game concept design is the light and shadow shapes, such as the original game concept design of character design, we can the application of the colour of this nature in relatively young feminine role, especially in depicting the role of color is most commonly used to. So depicting the face of relatively small, color of skin and excessive is soft and uniform; of course, we in the application to the scene, to the performance of some of the darker aspects of theme and must to add these elements, in close-up of some eerie and cold scene usually use hard light not too soft to, hard light to make the main character or scene surface looks clear in structure, to the shock strength. These are the experience accumulated in the usual hand drawing, in the original game concept design usually under the lighting and light how regulation can be only some simple to understand, in the characterization of as long as the picture effect should be expressed clearly on it, does not need, if this effect but not focus. The degree of fuzziness of the shadow of an object depends on the degree of light and shade, soft degree and high shadow picture, the soft and low shadow picture is clear, and the shadow can be changed from black to gray. The original game concept design use with layering and absolute texture character or scene, soft light frequent use. [3] 


\section{Game in the Brightness of the Light Source}

Game of the original painting in the light source brightness depending on the game itself to express the role object, high brightness is often used as a positive image expression, or masculine, just bright, bright and positive role in shaping, instead of the darkness of the larger image is often used as a negative, manifests the role, how to shape the more terrible, dark artistic effect, in a large extent is inseparable from the light, if these roles in later in the story changes, it is through the light of the connection and complete replacement of some.

\section{Pictures in the Shadow of Diminishing}

One of the properties of light and shade is often easily overlooked, it is the decline of light and shade, also known as attenuation. Attenuation in the picture I think this is the main light source to the surrounding objects emit light source through some delicate objects so that the light itself is subtle objects absorb reflection and refraction of a light decline phenomenon in the. Therefore, the attenuation of light can not be separated from the diffuse reflection, the two are a phenomenon of light and shadow produced alternately, so they are interrelated and need to be used in combination. So we look at some of the performance of the phenomenon. First of all, we should understand that the distance to the light intensity, which is due to the formation of the attenuation of light in the transmission process. Secondly, on the basis of this, we can show the effect of distance to achieve the unity of the picture itself.

The attenuation of light is inevitable, and it is also an important element to restrict the shadow. This is basic elements are summed up through the game's original designers, light attenuation is regular, but in a photography course, a general optical laws -- the inverse square law. The meaning of statements is mainly: exposure to light the surface distance directly restricts we feel the shadow of the present level, although we in the painting creation not directly apply these principles, but these must be constantly to be loaded in each creator's heart. The light area of the object should be small, and vice versa. By the light area is large, but we should also understand that some light larger and more dense distribution, the indirect reaction of the law of the second. We can show the distance of the object when the shadow, so that it becomes more blurred, so that can produce a meaning of attenuation. This is we in the original game concept design need to understand the theoretical knowledge of the master of the optical, knowledge to grasp the need to the test of practice, a start, how to put these elements refer to the original painting, which requires our bold despite constraints, tempted by optical. You will find that in fact in the game using the light attenuation in the still relatively frequent. For example, the original game concept design we often beating some of the roles of action, in this case shadow role itself requires using light attenuation performance. When the game characters to the ground contact, role playing games under the shadow will form a very sharp edge. This case we of the shadow is a black lump treatment is reasonable; game character jumps up, feet from the ground, the role itself under the shadow should produce light attenuation, the shadow itself should by deep to shallow a attenuation change. At the same time, on the edge of a shadow is bound to create fuzzy, most people in the process of practice but ignore the shadow attenuation changes in this vision, lost the details, will produce the illusion of the real scene. And excellent painting designers can seize the details, to further characterizations of the role of the game up to the landing between light and shade change, when landing in the shadow.

Draw more weakening, the use of a little bit of the gray shadow of the shadow of the additional blur, will make the game more details, And the work will be more vivid.

The character's face is very important in the design of the game, and the attenuation of the 
shadow of the face is very important to the work. Most of the original designers were able to take the initiative by shadow attenuation performance portray the character's faces, but not all are able to do this, after seeing a senior teacher of the original teaching materials, he is to elaborate such, when the main light source irradiation to the object light is strong, comparison contrast will become large, then in the original contrast between light and dark conditions will become more prominent. And when the main light is usually not strong situation, in the original game concept design reflected to the pale weak contrast and brightness of the shadow depends on the intensity of the light energy. So, the role of ear cheek shadow maps and scene around the role of light energy. But this situation in the game of the original painting of the junior staff often make such a mistake, master this rule but only single add stiff shadow, but paid no attention to the shadow in the image light source can combine and match.

Weather performance in game artwork will often see, specific game scenarios through the weather can play to heighten the link role. But the environmental performance in the original painting of the game screen is very difficult, and not all the games original artist are able to explain clearly. Here, we need to focus on is rain, smoke, water to the attenuation of a lot of light and shade, original designers need to clearly grasp the laws of Optics and the principle. The light travels in a very delicate space. And then to an object in the volume is small, then the light to walk and the continuous attenuation, but also need through the complex refraction, reflection and diffuse reflection, the beam is formed at this time there will be, the distance between dust objects will produce changes in different. In this case the need for game carvers to observe, through some material to find the change of optical photos. We can try some of the following performance. First, on the original character or scene were sketch rendering, determine the orientation of the body, especially in the center of the object positioning. Secondly, draw game scene and role of light by light roadmap; in the creation we first have to we know to the representation of the object need much energy to show. Underlie the association between the two. First, closer to the character or object, makes the picture from the near to the distant, giving the picture a sense of hierarchy, and the second is the picture thumbnail processing, clear light of the primary and secondary were reasonable allocation; finally strengthen the light and shadow, game scene showed some light beam, special effects, to enrich the picture. In reality, we observe the fog when Heaven penetrated light will appear to have a beam. This performance in line with the true nature of the law.

There is, the light attenuation is mainly used to show texture and sense of space, which is an important manifestation of attenuation. Because no matter what time there will be light and shadow of the decay of life, we can see everywhere. When you look up look up at the plane of the sky or HUD long-distance truck at the moment, will find that the farther the distance fuzzy bigger and bigger, the brightness will become increasingly weak. This is what we usually sense of light attenuation of reason. Clear these basic principles of optics, the game original artist can of distant objects are handling the weakened, to clear the attenuation laws of nature, but to meet the real laws of nature, too exaggerated lose authenticity, because the authenticity of the game screen is very important. This situation is mainly reflected in the process of changing the distance between the objects. It is important to apply the expression of light energy to the creation. Game scene in the original painting some darker scenes such as caves and cellars and caves, this kind of space are relatively strong game scenes need more light attenuation. If in a more closed room, at the same time the external environment is relatively dark. In this case will open at the top of the basement of the three windows, through the sunshine outdoor from three windows shines on performance of the basement of the sense of space, three beam of light through a number of gradient to show the effect, the indoor objects cannot have too much light effect using, then visual presentation will conflict, so 
you can according to the three light beam space and distance in length to reflect the color effect, can increase people's ability of spatial experience, create a very vast distance sense of space. In the space of the game scene, but also often used is the performance of the entire space scene only a weak light source. In the performance of such a scene, you can only depicted emphatically near the light source of the light attenuation transition effects and create the illusion of a deep space to the game player, in order to enrich the picture. Light attenuation is a phenomenon was evident in a normal life, careful observation of life and in game artwork drawn in practical application will help from the original design can accurately grasp the light attenuation is one of the most important rules. The need for special instructions, the Japanese style of painting in the game to simplify the shadow of this is also worth learning a little.

The completion of the production cycle, timeliness is also very important, the pursuit of a full picture of the shadow of the overall picture will cause the primary and secondary loss, or a waste of production cycle. Has formed the default shadows gamers visual senses, we can ignore to portray, can improve the working efficiency of the original, and shorten the production cycle. Give an example, game in Chinese style martial arts factions game character hair mostly with long hair, more elegant, the characterizations of not every ray shadows need light attenuation over, because the player most of the main effort invested in hair and dynamic elegant realized on the. Little attention to subtle changes in the shadow of the small problem. So in this case, we summarize the original game concept shadow attenuation performance of some rapid methods: have some very important shadows and foil atmosphere of the need in the shadow show, not necessary shadow is can save. In general this is several light attenuation in the design of the games. Need further explanation, we explained belongs to the creation of cohesion theory knowledge, the game artwork rendering to improve the role, but for most short time may not be immediate, but only after a lot of practice and accumulation and light attenuation constant practice and processing, then your game of the paintings will matter over.

\section{Color Light}

The concept of color in daily life is rarely mentioned, a subject which is the most commonly used professional photography. Photography in the described and compared in detail, color temperature from the word with temperature, but the academic did not temperature concept, it as interpretation of light color in receives light source objects forming and change. An exposure to normal color will have a standard color value, which is reflected in what the object receives light source. Cool colors of the formation is affected by light object color temperature value is higher than the general color temperature numerical, when a light object surface color temperature numerical below normal balance when the picture became red, color tends to warm. In other words, the color does not affect the brightness of the screen, but the color effect of the object itself. The reader will ask, color temperature in the original game concept design main role is that people usually under habit of color attributes are what? In the eyes of art design, the world consists of three colors: red, yellow, blue, and in the CG electronic painting and TV color display in the field, the three primary colors of light, as a picture of a standard color, in the world of color all colors are mixing three kinds of standard color and form a new color. Generally speaking, our eyes often feel green than any two colors to be bright, in fact, the gray level between the two used to replace the gray, the more dark some of the gray instead of blue. Understand the definition of color temperature value, it can probably pointed out sunny sky to give people the feeling is dark blue, candle in a dark place will show a yellow color. The color temperature of a role in the play.

In today's teaching and industry of game of the original painting, most important is by compliant 
or extensive expressive line, strong and hazy shadow and color mutual collocation ability reflected the, so in the performance of the original painting we want to put many of these elements are combined and synthetic physical distribution and utilization in order to reflect the good artistic effect.

The first step, the first to understand the light. Physics is the interpretation of the meaning of light, light is reflected by the expression of a kind of electromagnetic wave and the way of communication, it is a kind of virtual communication means to carry out the performance of the. Physics is called "the two wave of". Game design, use of light and shadow style is reflected in where?

The common meaning, because the light we can see real natural color, high light in Western painting art is refers to the object itself in the brightest point, after blocking the light, the surrounding light, and the object itself no shadow of, light shadowless body feeling bad, can think, Western painting art refers to the light is in fact our popular parlance of light and shade. [4]

Light is divided into five tone, high gloss, color transition, backlight, reflection and projection, these features is often used in our game painting creation of light and shadow shapes, because the quality of the game in continuous improvement, delicate picture degree in continuous improvement, soft light and in a great extent affects the details of the original game concept, how to grasp these delicate and soft lighting performance, is one of the most important factors need to be considered in the creation of the original games.

The boundary problem of light and shadow forms in the picture, can reflect the intensity and the permeability of light, in reality, in addition to the sun other industrial light source, all belong to soft light source. In the same time, soft is usually widely used in traditional painting and modern CG painting. An important branch of the use of light and shade in the original game concept design is the light and shadow shapes, such as the original game concept design of character design, we can the application of the colour of this nature in relatively young feminine role, especially in depicting the role of color is most commonly used to. So depicting the face of relatively small, color of skin and excessive is soft and uniform; of course, we in the application to the scene, to the performance of some of the darker aspects of theme and must to add these elements, in close-up of some eerie and cold scene usually use hard light not too soft to, hard light to make the main character or scene surface looks clear in structure, to the shock strength. These are the experience accumulated in the usual hand drawing, in the original game concept design usually under the lighting and light how regulation can be only some simple to understand, in the characterization of as long as the picture effect should be expressed clearly on it, does not need, if this effect but not focus. The degree of fuzziness of the shadow of an object depends on the degree of light and shade, soft degree and high shadow picture, the soft and low shadow picture is clear, and the shadow can be changed from black to gray. The original game concept design use with layering and absolute texture character or scene, soft light frequent use.

Game in the brightness of the light source. Game of the original painting in the light source brightness depending on the game itself to express the role object, high brightness is often used as a positive image expression, or masculine, just bright, bright and positive role in shaping, instead of the darkness of the larger image is often used as a negative, manifests the role, how to shape the more terrible, dark artistic effect, in a large extent is inseparable from the light, if these roles in later in the story changes, it is through the light of the connection and complete replacement of some.

Pictures in the shadow of diminishing. One of the properties of light and shade is often easily overlooked, it is the decline of light and shade, also known as attenuation. Attenuation in the picture I think this is the main light source to the surrounding objects emit light source through some 
delicate objects so that the light itself is subtle objects absorb reflection and refraction of a light decline phenomenon in the. Therefore, the attenuation of light can not be separated from the diffuse reflection, the two are a phenomenon of light and shadow produced alternately, so they are interrelated and need to be used in combination. So we look at some of the performance of the phenomenon. First of all, we should understand that the distance to the light intensity, which is due to the formation of the attenuation of light in the transmission process. Secondly, on the basis of this, we can show the effect of distance to achieve the unity of the picture itself.

Game development cannot be separated from the art people's cooperation, art images is very important for the subsequent development of the game as a whole. As most early and forefront of making parts, they were the first to come into contact with the entire game world to view the first batch of personnel, planning personnel art idea mainly dependent on the implementation of the original painting. In the original painting, props, scenes and characters are through in-depth communication with planning additional game art creators of creative to finish in the end, moreover, follow-up of $3 \mathrm{~d}$ production is mainly done through the concept design, finally completed the final image is introduced into the game big view of the world, art part of the game is completed. The development of computer science and technology is closely related to the development of the visuals of the game, is the rapid development and popularization of computer, computer prompted an increasing number of potential users, and the pursuit of better quality, visuals of the game is actually a combination of painting and art design. The common computer great creation function, a large number of the usage of material, comfortable and convenient environment and efficient design process, prompting visuals of the game rapid development, unlimited technique, delicate and exquisite picture, unique artistic characteristics of the artistic charm is unique to the visuals of the game.

The visuals of the game screen tone, light and shadow is one of the basic factors affecting the modelling, but it is the most intuitive. Light is often used in the original painting creation of the game, light and shadow have a larger impact on the image, affects the smooth of the picture, the appeal of emotion, image and so on, light and shadow to form shape is worthy of further research. In addition, the modelling of light and shadow is often overlooked in the design of the original game is an important element, it takes the form of light and shade layout to metaphor object of the game's strong sense of volume and picture. "Images of all present items need to be combined with the Angle of the light to be reconciled." Shadow "picture, with the continuous improvement of image quality is being game art research and development team, the head of the attention, because the light and shadow model directly affects the quality of the picture. Light and shadow in the modelling, the dark can nourish the picture restless mood, can be a game players feel the space of the extrusion and deep. Use the form of light and shadow, dynamic and static, weight, and so on change human visual concepts, create the aesthetic of a new kind of high level, enrich the image modelling, added new vitality for art, concept should be a game designer constantly explore the pursuit of goals. Shadow form the boundary problem of images is the strength of the light and the effect of permeability, in real life, in addition to the sun and other industrial sources, basically belongs to soft light. Light is downy usually were widely used in CG the traditional painting and modern painting. Visuals of the game in the design of light use is an important branch of the modelling of light and shadow. Light is divided into five main tone, specular, reflection and transition color, backlight, projection, these characteristics is our game original painting creation will use light and shadow in the modelling of expression, because the quality of the game is constantly improving, delicate degree is constantly improve, light soft degree to a great extent, affect the details of the visuals of the game, how to grasp the exquisite and soft light, visuals of the 
game is an important factor to consider in literary creation.

\section{The Color of the Light Source}

The color of light is the color of the light on the surface of the object, in daily activities, similar items, in the same light will show a different color transformation. For example, the sun, morning, noon, evening colors are not the same, the morning of yellow, wine red, noon to white, and the evening will be a red orange. Alternating seasons influence of light is large, the performance of different colors change, game in the world has the same season attributes in different seasons of the role attribute different must be after the partition, relatively strong summer light, light perception is relatively bright, but the relatively warm winter light, color is relatively strong, influence to the character and the scene itself will have very different, and will change the color of the object itself. Assuming a white object. At noon will performance white morning in the sunlight shining becomes pale yellow, in the evening show orange red, the moonlight at night shining presented gray blue. Can be explained, the color of the light directly to the game world and the role of the inherent color of the scene changes, the color of the world can not be the lack of light source color. The role of the more obvious, will make itself more bright and colorful picture.

\section{The Relationship Between Light and Shade}

In the ordinary life, there is a kind of frequently appeared in the shadow of the phenomenon of light is covered. The shadow of the role of shadow, props, use in numerous films and games of the paintings, use at most of the shielding case is: "shadow of the vampire." All kinds of demons in the screen before all is a shadow of terror as a prelude. And to show us a lot of children are not suitable to watch the scene or deliberately stressed that some of the dark shadow of the game, through the wall or other objects on the shadow to portray. This is a picture of the more commonly used to block a performance. Of course, shadow in game design is the most common form. Game or animation, the role or the shadow of the scene is not in place to deal with the lack of harmony, which first of all to the observer to violate the sense of life of common sense. Shadow figures or scenes of carrying the quality, if there is no shadow, the viewer will have a floating in the air is not practical sense, which do not meet the normal point of view, because in life the shadow everywhere. It has in the US. People have the habit of all objects with the shadow of visual rules, so inevitably the shadow starting center as the key observation found that in life people do not inadvertently will, the morning sunlight in his shadow is longer, at noon, when the shadow is the smallest, through the most simple common sense, people are not conscious of mastered shadow height and length determines the light intensity and range in any angle. The shadow does not exist, the human eye's reference standard will be lost, and thus will produce the illusion. Particularly interesting part, shadow in the original game concept and not our life that is living fine mapping. In the game, shadow generally use another meaning: the atmosphere, to express their inner feelings and so on.

Need to be paid attention to in the drawing process is million in the dark clouds of the sky, the shadow everywhere, the clear sky or lightning of night sky, often have very obvious shadow; haze of rain or dust fog weather will weaken the shadow contrast. It is directly related to the important role of light in the haze of the world, the weakening of light faster than the sunny day, so show the shadow of the comparison of the ambiguous. Game designers often by lengthening or shortening the shadow to guide the position of the sun light, innuendo expression with time in the game. More a kind of situation is the main characters of this game of the original painting dressed in different friezes, often use brim shadow to describe the role of mood, or hide the role of eyes and 
characterize demeanor. Shadow is widely used in the game world, the situation is complicated and changeable, every kind of method need small observations and the need to constantly in their original works in practice. So what is the main method of using game design in the light shade? Must be clear, realistic game technique is very difficult in the shadow. Because of the time requirements of the game art production cycle, so some of the shadow of the game art designers will be discarded to improve their work efficiency. [5]Examples show that drawing the picture should make the game character standing under the tree. Realism block must be the shadow of the leaves show portrayed in various roles on the. But this form of expression in the game art production is not allowed, this is a waste of time, and even if the performance is not so easy. Of course, is based on the image of the overall importance of the game scene to determine the accuracy of the shadow of the painting. If the scene only fleetingly, then shadow can choose not to appear, with some color with gray to regional performance, will also form a good visual effect. If the works must focus on rendering, and the need to present a more refined shadow effect, through our daily life pay more attention to the effect of shadow on the object, shadows are generally bump and shadow in the mapping to an object from the visual image is transform and chiaroscuro effect. May wish to stop the brush to find a shade of the trees were carefully observe and experience which the shadow, the shadow of some form of structure and wild gray painting line, see light movement after the shadow of how to change. Can also sit in a particular light to find inspiration. The main is to seriously take the initiative to analyze. After the analysis of the practice, shadow of reality in the shadow and we think there are more or less is not the same, grasp these differences, can change their work shadow method, make his paintings have their own unique. To improve the repair some real performance.

\section{References}

[1] Ma Jian-mei. Environmental art course teaching mode reform of professional landscape design [J]. Anhui Agricultural Science. 2011 (31)

[2] Tang Jun. The development of modern landscape design present situation and trend analysis [J]. Chinese Gardening Abstract. 2012 (9) : 97-2012.

[3] Han Yu. Experience type teaching, the landscape design course teaching practice research [D]. Nanjing: Nanjing College of Art. 2012

[4] Xiang Xiaoxiao. Environmental art professional teaching reform of landscape design study [J]. Journal of Nanchang Institute of Education. 2013 (4)

[5] liu Chun-bo . Analyses of landscape environmental art [J]. The Young Writer. 2010 (18) 